International Mathematical Forum, 2, 2007, no. 36, 1763 - 1766

\title{
The Exponential Map in Fundamental Locally Multiplicative Topological Algebras
}

\author{
E. Ansari-Piri ${ }^{1}$ and S. Ketabchi \\ Department of Mathematics, Guilan University \\ P.O. Box 1914, Rasht, Iran \\ eansari@guilan.ac.ir, sketabchi@guilan.ac.ir
}

\begin{abstract}
A class of topological algebras, which we call it a fundamental locally multiplicative one, has already been introduced and the exponential map has been defined before on complete metrizable fundamental locally multiplicative topological algebras. Here, in this note we prove the main identification about this map.
\end{abstract}

Mathematics Subject Classification: Primary 46H; Secondary 46A

Keywords: the exponential maps, the fundamental locally multiplicative topological algebras

\section{Introduction}

The meaning of fundamental topological algebras is initially introduced in [1]. The fundamental locally multiplicative topological algebra(abbreviated by FLM ) with a property very similar to normed algebras is also introduced in [4]. The fact that every locally bounded topological algebra is an FLM and an example of an FLM algebra which is not locally bounded and locally convex is considered in $[4,3]$.

In [2], and [4] some famous theorems from normed spaces and normed algebras have been extended to fundamental topological vector spaces and to FLM algebras. In [4], we also could define exponential map in complete metrizable FLM algebras.

In this note we follow the process of defining of exponential function on a complete metrizable FLM algebra and we prove the famous identification $e^{x+y}=e^{x} e^{y}$ for commutative elements $x$ and $y$ of the FLM algebras where this identity has been proved before only for Banach algebras [6].

\footnotetext{
${ }^{1}$ Corresponding author
} 


\section{The previous proved results and related def- initions}

In this section we have a collection of definitions and theorems which are all discussed in [1], [2], [3], and [4].

Definition 2.1 . Let $A$ be a topological vector space. We say $A$ is a fundamental topological vector space, if there exists $b>1$ such that for every sequence $\left(a_{n}\right)$ of $A$, the convergence of $b^{n}\left(a_{n}-a_{n-1}\right)$ to zero in $A$ implies that $\left(a_{n}\right)$ is Cauchy sequence.

Proposition 2.2 . Let $A$ be a fundamental topological vector space. Then, for every $c>1$ and every sequence $\left(a_{n}\right)$ of $A$, the convergence of $c^{n}\left(a_{n}-a_{n-1}\right)$ to zero in $A$ implies that $\left(a_{n}\right)$ is a Cauchy sequence.

Theorem 2.3. Suppose $b>1$ and $A$ is a fundamental metrizable topological vector space. Then $A$ is complete if and only if for every sequence $\left(x_{n}\right)$ with the condition $b^{n} x_{n} \rightarrow 0$ in $A$, the series $\sum\left(x_{n}\right)$ is summable.

Definition 2.4. A fundamental topological algebra is an algebra whose underlying topological vector space is fundamental .

Theorem 2.5 . Let $A$ be a complete metrizable fundamental topological algebra with unit, and $x \in A$. If for some $b>1, b^{n} x^{n} \rightarrow 0$ in $A$, then $1-x$ is invertible and

$$
(1-x)^{-1}=1+\sum_{n=1}^{\infty} x^{n} .
$$

Definition 2.6 . A fundamental topological algebra is called to be locally multiplicative, if there exists a neighborhood $U_{0}$ of zero such that for every neighborhood $V$ of zero, the sufficiently large powers of $U_{0}$ lie in $V$. We call such an algebra, an FLM algebra.

Definition 2.7 . Let $A$ be a topological vector space and let $E$ be any set. We say $A$ is uniformly fundamental on $E$, if there exists $b>1$ such that for every sequence $\left(f_{n}\right)$ of functions from $E$ into $A$, the uniform convergence of $b^{n}\left(f_{n}-f_{n-1}\right)$ to zero on E implies that $\left(f_{n}\right)$ is uniformly Cauchy on E. If $A$ is uniformly fundamental on every set $E$, then we call it a uniformly fundamental one.

Proposition 2.8. Let $A$ be a topological vector space. A is uniformly fundamental if and only if it is uniformly fundamental on $N$. 


\section{The main property of exponential map}

On a Banach algebra $A$ with unit, the definition of $\exp (a)$ for $a \in A$ is based as usual on the well-known theorem that: "the absolutely convergent series in Banach spaces are summable".

The theorem 2.3 extends this fact and proves it for complete metrizable fundamental topological vector spaces. Here, at first we use this result and recall the definition of exponential function on FLM algebras.

Let $A$ be a complete metrizable FLM algebra and $x \in A$. Let also $U_{0}$ satisfies in the definition of FLM (2.6), and $b>1$. There exists $\lambda>0$ such that $\lambda x \in U_{0}$ and so $\lambda^{n} x^{n} \rightarrow 0$. Since $\frac{b^{n} \lambda^{-n}}{n !} \rightarrow 0$, so $b^{n}\left(\frac{x^{n}}{n !}\right) \rightarrow 0$ in $A$. Now, by 2.3 the series $\sum_{n=1}^{\infty} \frac{x^{n}}{n !}$ is summable.

Definition 3.1 . Let $A$ be a complete metrizable FLM algebra with $1 \in A$ and let $a \in A$. Define $\exp (a)=1+\sum_{n=1}^{\infty} \frac{a^{n}}{n !} \in A$.

The fact that $\exp (0)=1$ and $\exp (-a) \exp (a)=1$ have been considered before [3]. Here we prove that for $x, y \in A$, when $x y=y x, \exp (x) \exp (y)=$ $\exp (x+y)$.

This is of course a well-known result of Banach algebras [5], and our proof is essentially based on what is done in [6] for Banach algebras, but here we do it in the absence of local convexity and local boundedness.

Definition 3.2 . Let $f:(a, b) \longrightarrow A$ be a map. We say $f$ is differentiable at $x_{0} \in(a, b)$ where as usual $\lim _{x \rightarrow x_{0}} \frac{f(x)-f\left(x_{0}\right.}{x-x_{0}}$ exists in $A$.

Proposition 3.3 . Let $A^{*}$ separates the points of $A$ and $f^{\prime} \equiv 0$ on $(a, b)$, then $f$ is constant.

Proof. Let $\phi \in A^{*}$ and $h:(a, b) \rightarrow C$ be defined by $h(x)=\phi o f(x)$. Then $h^{\prime}(x)=\phi\left(f^{\prime}(x)\right)=0$ and so $h$ is constant.

Proposition 3.4. Let $a \in A$ and $f: R \rightarrow A$ be defined by $f(t)=\exp (t a)$, then $f(0)=1$ and $f^{\prime}(t)=a f(t)$.

Proof. For $x \in A$, if we put $x^{0}=1$, then $f(t)=\sum_{n=0}^{\infty} \frac{t^{k} a^{k}}{k !}=\lim _{n \rightarrow \infty} s_{n}(t)$ where $s_{n}(t)=\sum_{k=0}^{n} \frac{t^{k} a^{k}}{k !}$. Then:

$$
s_{n}^{\prime}(t)=\sum_{k=1}^{n} k \frac{t^{k-1} a^{k}}{k !}=a \sum_{k=1}^{n} \frac{t^{k-1} a^{k-1}}{(k-1) !}=a \sum_{k=0}^{n-1} \frac{t^{k} a^{k}}{k !}=a s_{n-1}(t) .
$$


We claim $s_{n}$ converges uniformly to $f$ on any bounded subset of $R$ and so $s_{n}^{\prime}$ converges uniformly to af on such sets.

Since, $b^{n}\left(s_{n+1}(t)-s_{n}(t)\right)=\frac{b^{n} t^{n+1} a^{n+1}}{n+1}$ and $\frac{b^{n} M^{n+1} a^{n+1}}{n+1} \rightarrow 0$ in $A$, so if $t \in K$ then for $W \in N(0)$ and sufficiently large $n \in N$ :

$$
\frac{b^{n} t^{n+1} a^{n+1}}{n+1}=\frac{b^{n} M^{n+1} a^{n+1}}{n+1}\left(\frac{t}{M}\right)^{n+1} \in\left(\frac{t}{M}\right)^{n+1} W \subseteq W
$$

where $|t| \leq M$ on $K$. Therefore $\left(s_{n}\right)$ and $\left(s_{n}^{\prime}\right)$ converges uniformly on $K$ and hence $f^{\prime}(t)=a f(t)$.

Theorem 3.5 . Let $g: R \rightarrow A$ be any map such that $g(0)=1$ and $g^{\prime}(t)=$ ag $(t)$ for a fixed $a \in A$, and $f: R \rightarrow A$ be defined by $f(t)=\exp ($ ta $)$, then $g \equiv f$ on $R$.

Proof. Define $h: R \rightarrow A$ with $h(t)=f(t) g(-t)$. Now, since $a f(t)=$ $f(t) a, h^{\prime}(t)=f(t)^{\prime} g(-t)-f(t) g^{\prime}(-t)=a f(t) g(-t)-f(t) a g(-t)=0$. Hence, for all $t \in R, h(t)=h(0)=1$. Therefore $e^{-t a} g(t)=1$. Now, since for all $x \in A, \exp (x) \exp (-x)=1$, we get $g(t)=f(t)$.

Theorem 3.6. For $a, b \in A$ when $a b=b a \exp (a+b)=\exp (a) \exp (b)$.

Proof. Let $f: R \rightarrow A$ be the map with $f(t)=\exp (t a) \exp (t b)$ then, $f^{\prime}(t)=a \exp (t a) \exp (t b)+\exp (t a) b \exp (t b)=(a+b) f(t)$. Therefore $f(t)=$ $\exp (t(a+b)$, and $\exp (a+b)=\exp (a) \exp (b)$.

Corollary 3.7. The set $\operatorname{Exp}(A)=\{\exp (a): a \in A\}$ is a group.

\section{References}

[1] E. ANSARY-PIRI, A class of factorable topological algebra, proceeding of the Edinburgh Mathematical Society (1990) 33, 53-59.

[2] E. ANSARY-PIRI, Completion on fundamental topological algebras, Honam Mathematical J.26(2001), No.1, 79-83.

[3] E. ANSARY-PIRI, The linear functionals on fundamental locally multiplicative topological algebras, submitted.

[4] E. ANSARY-PIRI, Topics on fundamental topological algebras, Honam Mathematical J.23(2001), No.1, 59-66.

[5] F.F. BONSALL and DANCAN, Complet normed algebras, Berlin (1973).

[6] G.J. Morphy, $C^{*}-$ algeras and operator therory, ACADEMIC PERESS, INC.

Received: November 1, 2006 